This item was submitted to Loughborough's Research Repository by the author.

Items in Figshare are protected by copyright, with all rights reserved, unless otherwise indicated.

\title{
Characterisation and numerical modelling of complex deformation behaviour in thermally bonded nonwovens
}

PLEASE CITE THE PUBLISHED VERSION

http://dx.doi.org/10.1016/j.commatsci.2013.01.007

\section{PUBLISHER}

(C) Elsevier B.V.

VERSION

AM (Accepted Manuscript)

\section{PUBLISHER STATEMENT}

This work is made available according to the conditions of the Creative Commons Attribution-NonCommercialNoDerivatives 4.0 International (CC BY-NC-ND 4.0) licence. Full details of this licence are available at: https://creativecommons.org/licenses/by-nc-nd/4.0/

\section{LICENCE}

CC BY-NC-ND 4.0

\section{REPOSITORY RECORD}

Farukh, Farukh, Emrah Demirci, Baris Sabuncuoglu, Memis Acar, Behnam Pourdeyhimi, and Vadim V. Silberschmidt. 2019. "Characterisation and Numerical Modelling of Complex Deformation Behaviour in Thermally Bonded Nonwovens”. figshare. https://hdl.handle.net/2134/16995. 
Characterisation and Numerical Modelling of Complex Deformation Behaviour in Thermally Bonded

Nonwovens

Farukh Farukh ${ }^{(1)}$, Emrah Demirci $^{(1)}$, Baris Sabuncuoglu ${ }^{(1)}$, Memiş Acar ${ }^{(1)}$, Behnam Pourdeyhimi $^{(2)}$, Vadim V. Silberschmidt ${ }^{(1)}$

1 - Wolfson School of Mechanical and Manufacturing Engineering, Loughborough University, UK

2 - Nonwovens Cooperative Research Center, North Carolina State University, Raleigh, NC, USA

*Corresponding author’s e-mail: F.Farukh@lboro.ac.uk

Tell: +441509227621 ; fax: +441509227585

Add: Wolfson School of Mechanical and Manufacturing Engineering, Loughborough University, UK

Keywords: nonwoven; polypropylene; viscous; finite element; damage

\begin{abstract}
:
A complex time-dependent deformation and damage behaviour in polymer-based nonwovens are analysed under conditions of multi-stage uniaxial loading. Elastic-plastic and viscous properties of a polypropylene-based fabric are obtained by series of tensile, creep and relaxation tests performed on single fibres extracted from the studied fabric. These properties are implemented in a finite-element (FE) model of nonwoven with direct introduction of fibres according to their actual orientation distribution in order to simulate the rate-dependent deformation up to the onset of damage in thermally bonded nonwovens. The predictions of FE simulations are compared with the experimental data of multi-stage deformation tensile tests and a good agreement is obtained including the mechanisms of deformation. Due to direct modelling of fibres based on their actual orientation distribution and implementation of viscous properties, the model could be extended to other types of polymer-based random fibrous networks.
\end{abstract}

\title{
1 Introduction
}

Nonwovens and their combinations with other materials are used in numerous applications, including geotextiles for soil reinforcement, medical applications, filtration, and aerospace industry [1]. Regardless of the application area of nonwovens, understanding of their deformation and damage behaviour, as well as of relationships between their macroscopic mechanical behaviour, underlying fibre properties and randomness of their microstructure can help to design and optimise the performance of such materials.

Nonwoven fabrics are generally made from continuous or staple fibre webs strengthened by bonding using various techniques such as mechanical bonding, bonding by adhesive or thermal bonding [1]. Compared to other bonding processes, thermal bonding and the products made with this technique offer several advantages such as lower capital investment and manufacturing cost, higher throughput, no hazardous chemical involved and very good 
recyclability $[1,2]$. Therefore, it is one the most commonly used technique for the manufacture of nonwovens [3]. In thermally bonded nonwovens, fibrous web is passed through a hot calendar with an embossed pattern. Bonding mainly occurs at raised areas resulting in bonded spots called "bond points". On the other hand, web regions, which are not in contact with the hot engraved pattern, remain unaffected and form the fibre matrix that acts as a link between the bond points. The structure of resulting thermally bonded nonwoven (shown in Fig. 1) is a combination of continuous and discontinuous regions, thanks to which it exhibits a unique and complex mechanical behaviour. The understanding of deformation and damage behaviour of this type of material will help to improve design of products aimed at higher reliability and durability.

A polypropylene-based low-density thermally bonded nonwoven is studied in this work. A deformation and damage behaviour of low-density nonwovens is complicated due to randomness, discontinuity and presence of voids in their microstructure. Moreover, polymerbased constituents exhibit a nonlinear elastic-plastic behaviour including viscous effects, which is the most significant property of polymer materials. Due to these viscous properties, polymer-based materials demonstrate a rate-dependent behaviour such as stress relaxation, creep and damage. Several studies have been conducted to predict the behaviour of such materials by using various numerical modelling techniques. Most of these studies provide a partial solution to the problem of predicting the changes in mechanical behaviour with changes in orientation of constituent fibres [4-6], curliness of fibres [7] and manufacturing conditions [8]. A very few studies were performed to develop a finite element model that can simulate the response of nonwoven materials under mechanical loading [9-17]. All of these studies either focused on initial deformation of the material without considering damage or included only elastic-plastic material properties. To author's knowledge, none of these models can predict the damage behaviour of nonwovens while incorporating material's viscous properties. The viscous properties of constituent fibres have a significant effect on the material's behaviour e.g. load-rate sensitivity, stress relaxation and creep-based deformation and damage. Generally, nonwovens in their service life undergo complex loading at various strain rates with or without effects of creep or stress relaxation rather than simple monotonic loading and damage occurs inevitably in the material beyond a certain level of loading. Thus, it is important to understand the damage mechanisms that govern the material behaviour under excessive loading and to develop a finite-element model incorporating the actual orientation distribution and viscous properties of constituent fibres capable to reproduce the response of these materials - including their damage - for complex loading histories.

A finite-element model based on nonwoven's microstructure and properties of constituent fibres (including viscous ones) will provide insights in, and better exploration of, the design space of products containing nonwoven parts, which is the aim of this study. A parametric finite-element modelling technique is used to incorporate the orientation distribution of fibres, with the number of fibres exactly equal to that in the actual fabric. Using this parametric technique, randomness of the fabric's structure is captured by direct modelling of fibres and thus explicitly accounts for the main mechanisms of deformation (affected by time-dependent parameters of constituent fibres) of thermally bonded nonwoven material up to the onset of damage. Elastic-plastic and viscous material properties, considered significant in case of polymer-based nonwovens and obtained by performing a series of experiments on constituent fibres, are implemented in the model. The results obtained with the model were compared with those of multi-stage tensile loading tests on nonwovens in two principal directions: machine direction (MD) and cross direction (CD) up to the onset of damage. 


\section{Experimentation}

\subsection{Material}

A polypropylene (PP) based mono-component thermally bonded low-density nonwoven was used in this study. Its basis weight was $20 \mathrm{~g} / \mathrm{m}^{2}$ and it was manufactured at an optimal bonding temperature between $150^{\circ} \mathrm{C}-160^{\circ} \mathrm{C}$. Randomly oriented fibres were thermally bonded with square-shaped bond points (Fig. 1). Approximately $14 \%$ of the fabric area was bonded with bond density of 23.68 spots $/ \mathrm{cm}^{2}$.

\subsection{Single-fibre behaviour}

Nonwoven fabric used in this study was composed of PP fibres having a length, diameter and linear density of $38.1 \mathrm{~mm}, 18 \mu \mathrm{m}$ and 2.3 denier, respectively. Fibres were extracted from a free edge of the fabric, and a series of experiments were performed to obtain the required material properties. Both edges of extracted fibres were attached to the sticky strips of paper to prevent slippage, dislocation and damage, and to provide a firm grip and convenient mounting on Instron ${ }^{\circledR}$ Micro Tester having $\pm 5 \mathrm{~N}$ load cell. A high-speed camera (Photron Fastcam SA $3^{\circledR}$ ) was used to make sure that fibres were not stretched during preparation and mounting of single-fibre specimens. Tensile, creep and relaxation tests were performed on such specimens at various loading rates, levels of engineering stress and strain. All the parameters necessary to model the elastic-plastic behaviour of a fibre such as its modulus of elasticity and flow curve were obtained with the uniaxial tensile tests whereas its viscous properties were quantified through the creep and relaxation tests. The details of single-fibre tensile test are given elsewhere [17]. The viscous properties obtained with single-fibre creep tests were implemented into the FE model based on a creep strain rate as a function of time and stress level (Fig. 2). Curve-fitting procedures were carried out to obtain smooth curves for a creep strain rate as a function of time and stress level [13]. Generally, creep tests of ordinary materials are performed at stress levels below the yield stress of the material; however, the model in this study was developed to simulate deformation up to the onset of damage. At this stage most fibres bear stresses significantly higher than yield stress; therefore, single-fibre creep tests were performed at various stresses including those exceeding yield stress (75MPa in this case). Since creep in material can cause deformation ultimately leading to material damage, all three stages of creep - primary, secondary and tertiary - were implemented in the model. However, due to limitations of the test rig including its low response time, creep tests covering also a tertiary stage were performed at stresses higher than $20 \mathrm{MPa}$. At stresses below this, single-fibre creep tests were performed or max. $180000 \mathrm{~s}(50 \mathrm{~h})$. Single-fibre relaxation tests were used for validation of the material properties implemented in the finite-element model.

\subsection{Fabric's behaviour}

Mechanical performance of a $20 \mathrm{~g} / \mathrm{m}^{2}$ thermally-bonded nonwoven composed of PP fibre was studied with multistage uniaxial tensile tests. The machine used for this purpose was Instron ${ }^{\circledR}$ Micro Tester with a $5 \mathrm{~N}$ load cell. Photron Fastcam SA3 ${ }^{\circledR}$ was also used to ascertain the evolution of deformation and damage mechanisms in thermally bonded nonwovens. The full details of the setup are given in [18]. These tests revealed that fibres started to re-orient and straighten themselves in the loading direction immediately at the beginning of tension. Once fibres were aligned along the loading direction, they underwent large elastic-plastic deformation and the maximum load was attained under this condition before the onset of damage. The observed damage initiation in the fabric was in a form of progressive failure of 
fibres. The load was recorded continuously together with a cross-head displacement during the tests. In order to observe the effects of viscous properties, tensile tests were performed with a complex loading history (strain rate was changed step by step accompanied also by stress relaxation) rather than as monotonic loading at constant strain rate (see experimental graphs in Fig. 8). This type of loading helps to observe the deformation behaviour of nonwoven under conditions close to those of their life in service where the fabric can undergo various combinations of tensile, creep and stress relaxation stages depending upon its use. Hence, specimens oriented in MD and CD were tested up to the onset of damage. It was observed that a strain-rate effect on the fabric's response was significant as it became less stiff with a decreasing strain rate and significant stress relaxation. Moreover, it was found that the results for mechanical properties were significantly different in both orientations - MD and $\mathrm{CD}-$ as shown in experimental graphs of Fig. 8.

\subsection{Structural characterization}

Mechanical anisotropy observed as a direction-dependent mechanical behaviour is the most prominent feature of nonwoven materials. This phenomenon is induced by a preferential orientation distribution with more fibres oriented towards MD. This orientation distribution of fibres must be considered to provide realistic simulations of deformation and damage behaviours of nonwovens including anisotropy since it plays a vital role in characterization of their mechanical properties. In order to obtain the orientation distribution of fibres in the fabric, micro-scale images were taken using scanning electron microscopy (SEM) and X-ray micro computed tomography $(\mathrm{mCT})$. The image with a clearest view of fibres was processed with the help of in-house software Nonwoven Anisotropy VI (see [18]). The software presented results in terms of orientation distribution function (ODF) of fibres as a histogram; details of this process are given in [18]. The obtained ODF was implemented into the finiteelement model. Information about structural entities such as dimensions, geometry and pattern of bond points (given in Table 1) was also obtained from the SEM images of the fabric.

\section{$3 \quad$ Finite-Element Model}

Finite-element simulations of the material were carried out using MSC. Marc ${ }^{\circledR} 2012$ as solver and MSC. Patran ${ }^{\circledR}$ as pre-processor. The simulations were performed within the framework of quasi-static deformation with large displacement and rotation considering the non-stressed state as reference. In simulations of nonwoven materials, it is challenging to introduce realistic randomness of material's microstructure in a FE model because of a large number of fibres to be modelled. Moreover, it makes it difficult to ensure that all the fibres are connected to bond points at an angle according to ODF suitable for the uniform meshing of the structure. All these issues were resolved by employing a parametric modelling technique in this study [13]. A special subroutine was written employing Patran Command Language (PCL) that contained all the information about the model including the orientation distribution and number of fibres, length of a single fibre, size, shape, and pattern of bond points obtained from the experimentation. MSC. Patran can read this code and compute the model incorporating the actual orientation distribution of fibres in terms of the experimentally obtained ODF. The number of fibres to be modelled was calculated by the subroutine using the following relation: 


$$
N F=\frac{F D \rho_{\text {Fabric }}}{\rho_{\text {Fibre }} L_{\text {Fibre }} C A},
$$

where $F D$ represents the area of the fabric to be modelled, $\rho_{\text {Fabric }}$ is the density of the fabric (20 g/m $\mathrm{m}^{2}$ in this study), $C A$ is the fibre cross sectional area, $\rho_{\text {Fibre }}$ is the density of fibre material and $L_{\text {Fibre }}$ is the length of the staple fibre. This calculation is based on the assumption that all the fibres have an equal and constant cross-sectional area along their length. The subroutine reduces significantly the effort required to reformulate the FE model to incorporate variations in ODF or other geometrical properties, which is a distinguishable feature of this modelling technique.

The FE model for the nonwoven under study was composed of continuous and discontinuous regions called bond points and matrix, respectively (Fig. 1). Both regions have distinct microstructures and were modelled using shell elements (element type 139 in MSC. Marc®) for bond points and truss elements (element type 9 in MSC. Marc ${ }^{\circledR}$ ) for fibres [20]. Truss elements do not carry any bending load, thus represent adequately the response of real fibres. Since nonwovens are anisotropic materials, two FE models of thermally bonded nonwovens were developed using the subroutine to simulate the behaviour of the material along MD and CD. Models with dimensions $16.5 \mathrm{~mm}$ x $10 \mathrm{~mm}$ were developed, with a higher dimension parallel to MD or CD. The geometry and the pattern of bond points in the model were identical to that of the fabric (Fig. 4) and given in Table 1. The thickness of bond points $(0.0337 \mathrm{~mm})$ and the diameter of an individual fibre $(0.018 \mathrm{~mm})$, measured with X-ray micro CT specimen, were assigned to corresponding elements. In order to check the model's validity with regard to force-displacement results, the boundary conditions implemented in the model were similar to those used in tensile tests as a combination of stages at various strain rates as well as stress-relaxation (Fig. 3). These conditions were applied on AB and YZ sides of the model (Fig. 6). The material properties implemented in the model were validated first by performing a single-fibre simulation. The results obtained from that simulation were then verified by comparing them with the data of single-fibre relaxation experiments as shown in Fig. 5. These validated material properties were then implemented into the developed FE model.

\section{Results and Discussion}

In order to replicate the experiments, a full specimen was simulated, using the same dimensions, aspect ratio, boundary conditions and rate of extensions as in experiments. The results of simulations were compared with the experimental data with regard to forcedisplacement curves as shown Fig. 8. The behaviour of material up to the onset of damage is well captured for both MD and CD directions including the load jumps caused by step decreases in the strain rate. Due to complex microstructure of nonwovens, several mechanisms are involved in their deformation, which act simultaneously, such as large rotation and straitening of fibres in loading direction, as well as failure at their stress or strain threshold [18]. All these mechanisms and their interaction up to the point of the onset of damage can be analysed with this FE model. The variation in stress-strain response with the specimen's orientation as a result of orientation distribution of fibres demonstrates that the model can well capture the material's anisotropic behaviour. 
A slightly stiffer initial behaviour - as compared to the tests - was found in the numerical results, which can be attributed to the fact that the curliness of fibres is not considered. The analysis of SEM image shown in Figs. 1 and 4a indicates that fibres in the fabric are not straight but curled. They only participate in load bearing when they are fully straightened under axial tensile stretching. Since the fibres in the FE model were not curled, they started bearing the load immediately after reorientation in the loading direction, leading to a stiffer behaviour of fabric at the beginning of mechanical loading. The fabric was extended up to strain of 0.5 corresponding to the damage initiation as observed in experiments. In order to simulate the damage evolution until failure of the fabric, criteria for a single-fibre failure should be implemented into the model. The development of these criteria for single-fibre failure is a significant experimental challenge due to issues of handling of specimens because of softness and size of the fibres. These fibres failure criteria will be developed and introduced at the next stage of investigation. As can be observed in Fig. 6, all the fibres are not at the same level of stress in any particular direction. Since the numerical model in this study is developed with direct introduction of fibres according to their orientation distribution within the fabric, it can predict the spread in stresses in members of the network structure. The distribution of the stresses in fibres at fabric's extension of 50\% is given in Fig. 7 for both MD and CD. Moreover, the deformation pattern predicted by the model was similar to that observed for the fabric as obvious in Figs. $6 \mathrm{c}$ and d; diamondshaped pattern arises during deformation of the fabric in CD.

The global shapes of the deformed specimens in simulations were compared with experimental images of fabric for both $\mathrm{CD}$ and MD as shown in Fig. 6. These shapes were quite similar to those from experiments at any level of deformation. It indicates that the model is capable to simulate the effect of grips, giving the maximum transverse contraction at the specimen's centre since it was restricted by the rubber grips at the edges of the fabric specimen. Besides, the mechanical behaviour and anisotropy of nonwoven predicted by the model was similar to the one observed in experiments (Fig. 8).

\section{Conclusions}

In this study, complex deformation of low-density nonwovens was analysed with a parametric computational model incorporating elastic-plastic and viscous properties of the constituent fibres. FE simulations were implemented by direct introduction of fibres, equal in number to those of the fabric and with the same dimensions, into the model. The fibres were introduced into the model according to their orientation distribution measured for the real fabric. Several advantages can be attributed to direct fibre modelling, e.g. natural account for voids and gaps in the microstructure as well as mechanisms involved in deformation and damage, making it more adequate for simulations of low-density nonwovens. It was shown that the model was not only capable to simulate the mechanisms involved in deformation up to damage initiation, material's anisotropy, effect of constraints due to grips and monotonic stress-strain behaviour accurately but it also captured the load jumps (step increase or step decrease) when the tensile strain rate was changed. Since fibres were modelled directly using 
a parametric modelling technique to capture the network structure, it is expected that the approach will unveil the behaviour and mechanisms involved in damage initiation and evolution with changes in network topology. Since the model can predict the stress level in each element of the fibre network, it can help to find out the areas where the damage will initiate. Besides, thanks to incorporation of elasto-visco-plastic properties of constituent fibres; the model can be extended beyond the analysis of effects of variation in basis weight, material properties of the constituent fibres and dimensions of structural entities to predict the mechanical behaviour of other types of fibrous networks. A numerical tool to for analysis of nonwoven networks with a number of fibres equal to that for the actual fabric and accounting for their realistic orientation distribution and elasto-visco-plastic properties is developed for the first time. It can be used to assess the behaviour of nonwovens not only under monotonic loading but multi-stage loading regimes including stages of creep and stress relaxation and can help the industry to reduce significantly the use of trial-and-error approach in design and development of products containing nonwoven parts.

\section{Acknowledgement}

We greatly acknowledge support by the Nonwovens Cooperative Research Centre of North Carolina State University, Raleigh, USA. FiberVisions ${ }^{\circledR}$, USA generously provided the material for this study. We also acknowledge the use of Photron (Fastcam SA3), borrowed from the EPSRC UK Engineering Instrument Pool.

\section{References}

[1] W. Albrecht, H. Fuchs, W. Kittelmann, Nonwoven Fabrics: Raw Materials, Manufacture, Applications, Characteristics, Testing Processes, Wiley-VCH, Weinheim, 2003.

[2] S. J. Russell, Handbook of Nonwovens, Woodhead Publishing Limited, 2007.

[3] S. Michielsen, B. Pourdeyhimi, P. Desai, J. Appl. Polymer Sci. 99 (5) (2006) 2489-2496.

[4] S. Adanur, T. Liao, Textile Res. J. 69(11) (1999) 816-824.

[5] H.S. Kim, B. Pourdeyhimi, J. Textile Apparel Tech. Manag. 1 (4) (2001) 1-7.

[6] H.S. Kim, Fibers and Polymers 5 (2) (2004) 139-144.

[7] A. Rawal, J. Indus. Textiles 36 (2) (2006) 133-149.

[8] S.B. Gajanan, K.J. Praveen, E.S. Joseph, J. App. Poly. Sci. (2004) 92 3593-3600.

[9] E. Demirci, X. Hou, M. Acar, B. Pourdeyhimi, V.V. Silberschmidt, Solid State Phen. (2012) 188:164-169.

[10] E. Demirci, M. Acar, B. Pourdeyhimi, V.V. Silberschmidt, Appl. Mech. Mater. (2011) $70: 410-415$. 
[11] E. Demirci, M. Acar, B. Pourdeyhimi, V.V. Silberschmidt, Comput. Mater. Sci. (2011) 50:1286-1291.

[12] E. Demirci, M. Acar, B. Pourdeyhimi, V.V. Silberschmidt, ASME Conf Proc; ESDA (2010): 117-122.

[13] B. Sabuncuoglu, M. Acar, V.V. Silberschmidt, Comput. Mater. Sci. (2012) 52:164-170.

[14] X. Hou, M. Acar, V.V. Silberschmidt, Comput. Mater. Sci. (2011) 50:1292-1298.

[15] X. Hou, M. Acar, V.V. Silberschmidt, J. Mater. Sci. (2011) 46:307-315.

[16] X. Hou, M. Acar, V.V. Silberschmidt, Comput. Mater. Sci. (2009) 46:700-707.

[17] F. Farukh, E. Demirci, B. Sabuncuoglu, M. Acar, B. Pourdeyhimi, V.V. Silberschmidt, Comput. Mater. Sci. (2012) 64, 112-115.

[18] F. Farukh, E. Demirci, M. Acar, B. Pourdeyhimi, V.V. Silberschmidt, J. Mat. Sci. (accepted).

[19] E. Demirci, M. Acar, B. Pourdeyhimi, V.V. Silberschmidt, Comput. Mater. Sci. (2012) 52:157-163.

[20] Marc, Volume B, Release 2012, Element Library. 


\section{Figure Captions}

Fig. 1. SEM image of nonwoven fabric composed of bond points and fibre matrix

Fig. 2. Single-fibre creep tests for various stress levels

Fig. 3. Loading conditions in simulations including stages at various strain rates and stress relaxation

Fig. 4. (a) SEM image of $20 \mathrm{~g} / \mathrm{m}^{2}$ nonwoven material. (b) Corresponding part of FE model

Fig. 5. Experimental and numerical results for single-fibre relaxation at strain of 0.8

Fig. 6. Results for deformed nonwoven after 50\% extension: experiment (a) and simulations (b) in $\mathrm{MD}$; experiment (c) and simulations (d) in $\mathrm{CD}$

Fig. 7. Stress distribution in fibres at fabric's extension of 50\% in MD (a) and CD (b)

Fig. 8. Experimental and numerical force-extension curves for MD (a) and CD (b) showing jumps (step decreases) with changing strain rate 
Table 1. Parameters of geometry and pattern of bond points (BPs)

\begin{tabular}{llllllll}
\hline $\begin{array}{c}\text { Fabric's } \\
\text { orientation with } \\
\text { reference to BP }\end{array}$ & BP pattern & A & B & C & D & E \\
\cline { 3 - 7 } & & & & & & & \\
\hline
\end{tabular}




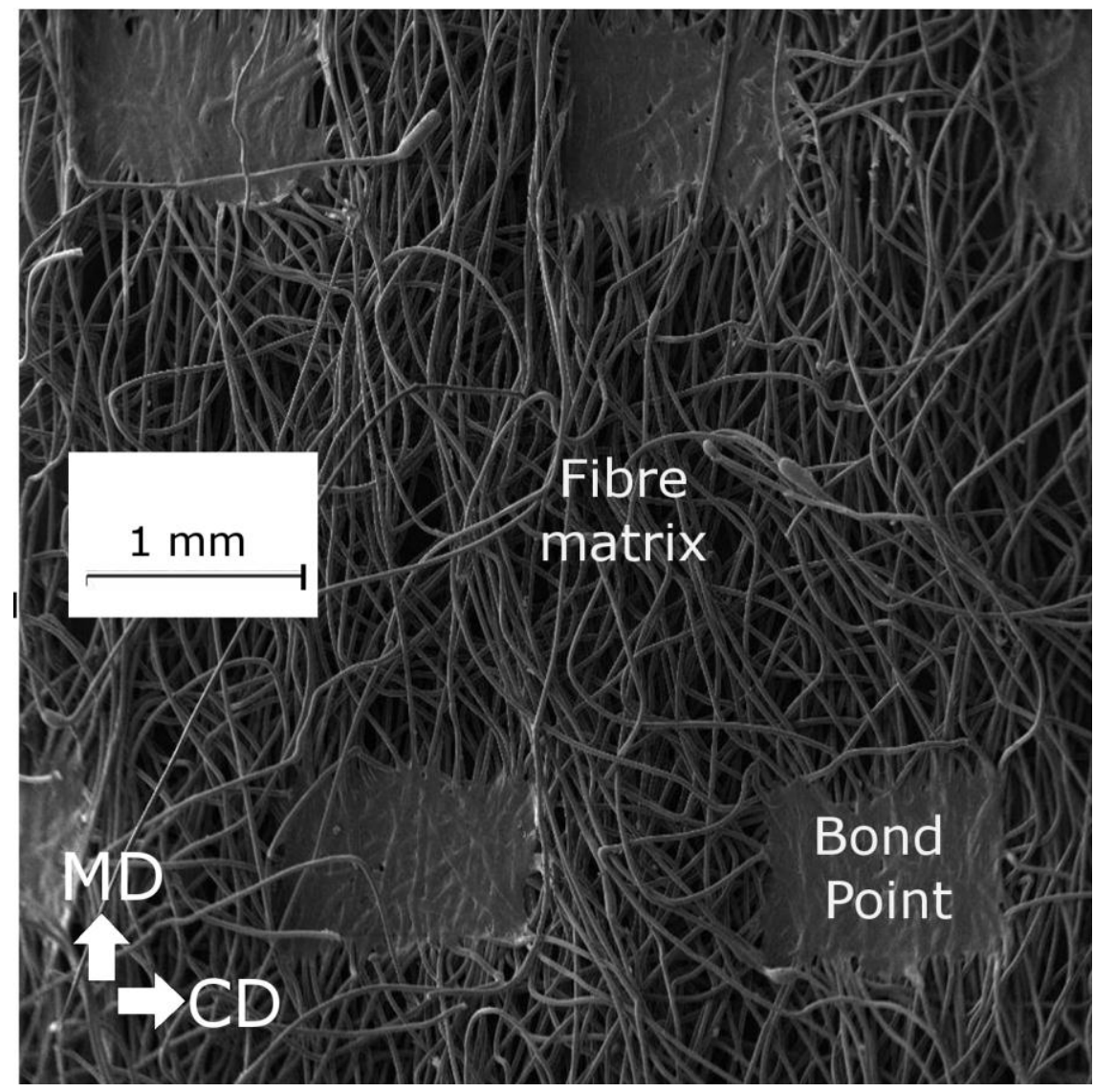

Fig. 1 


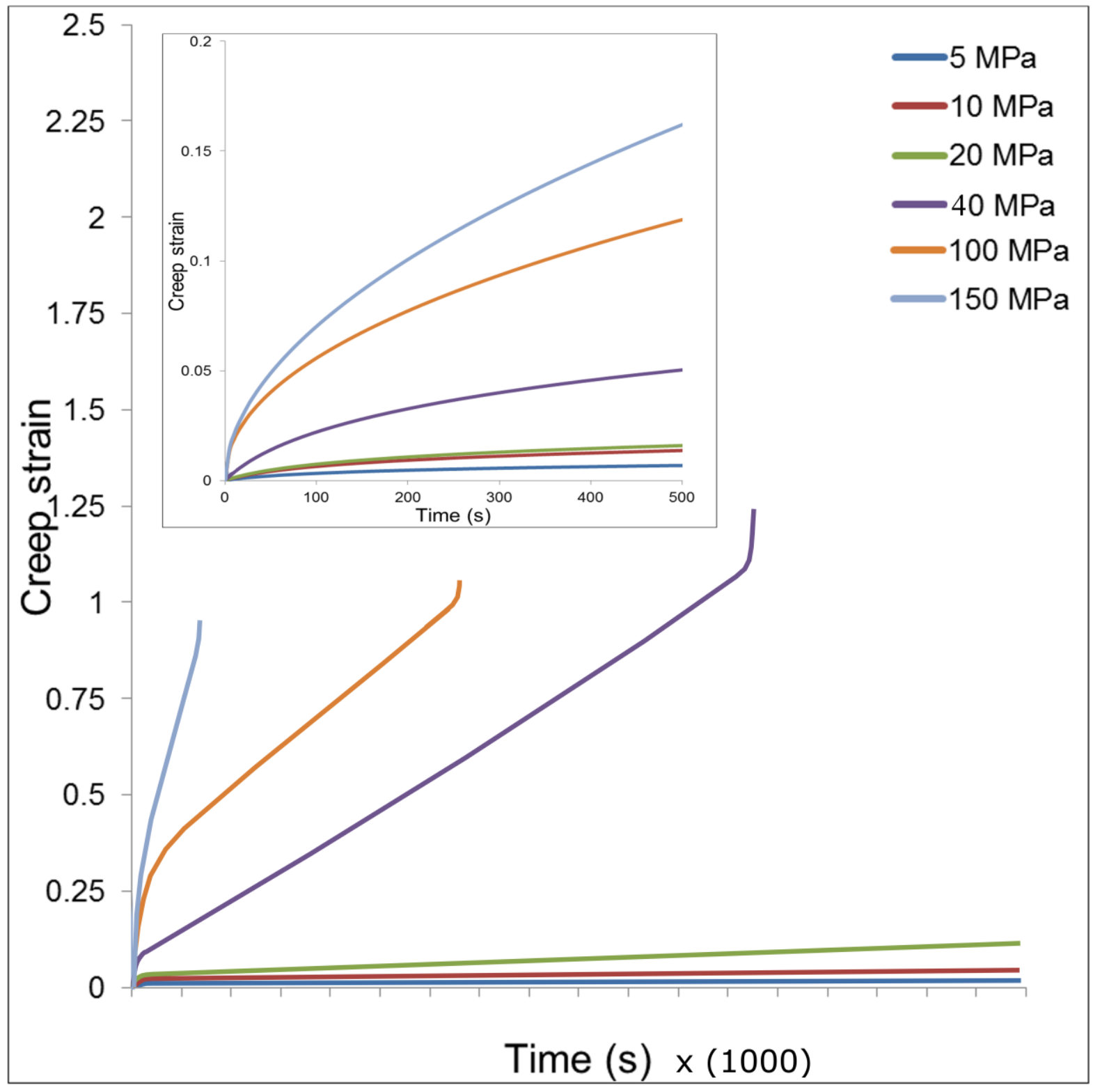

Fig. 2 


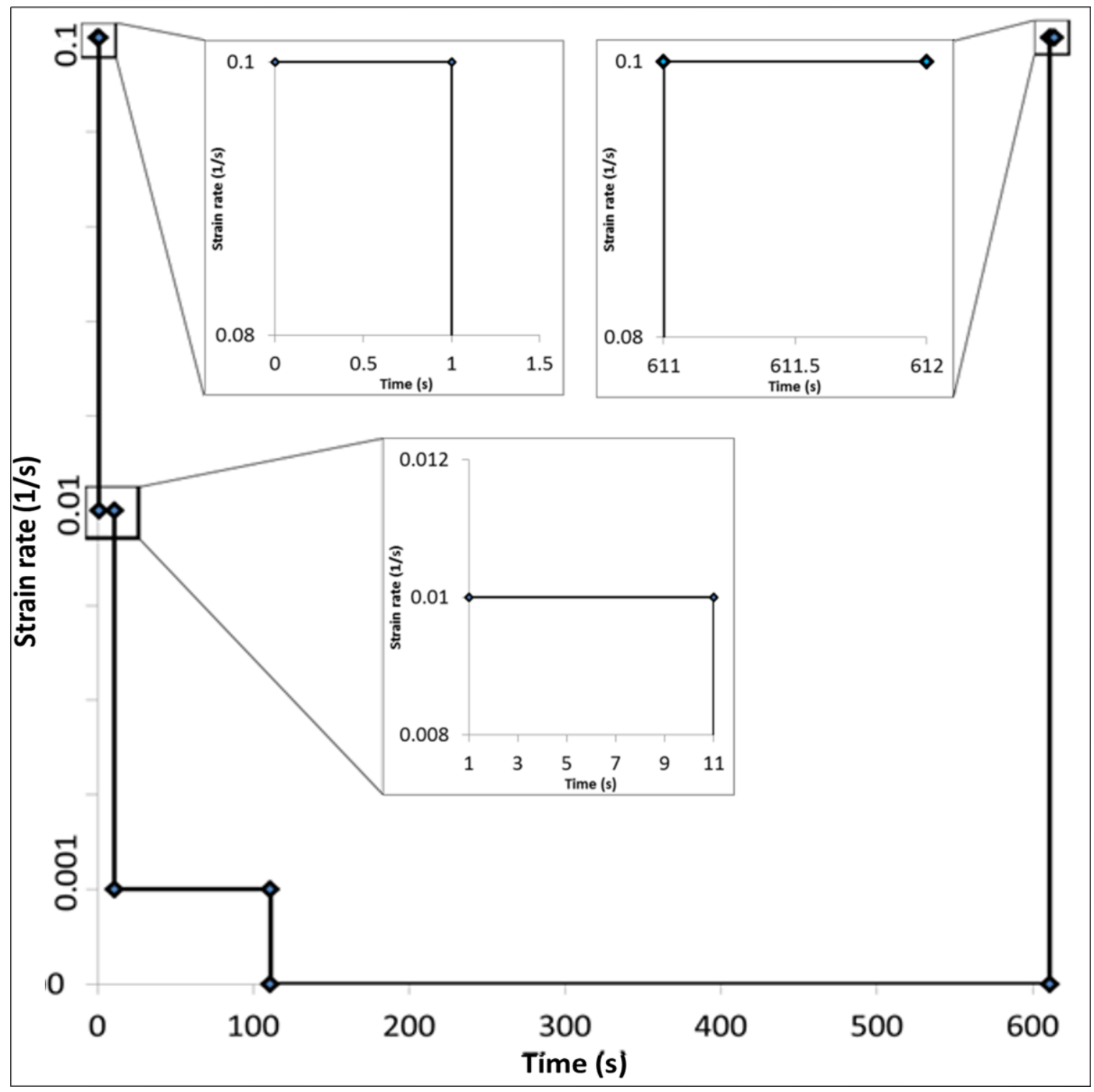

Fig. 3 


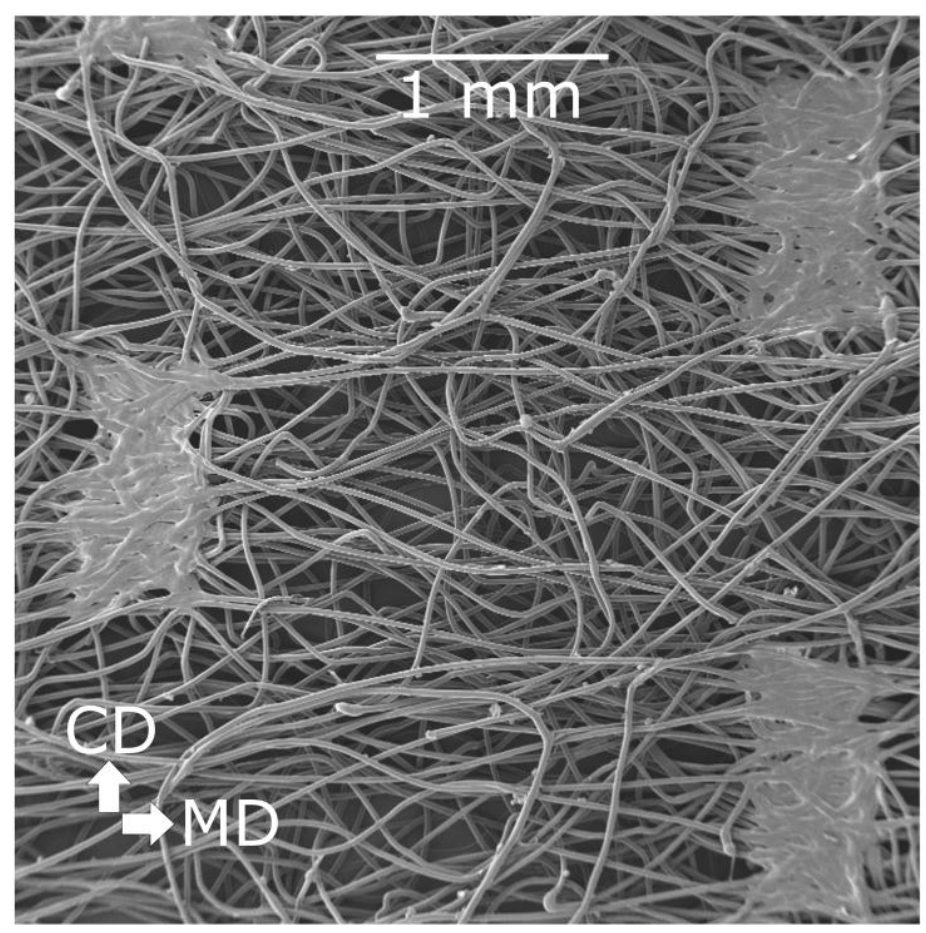

(a)

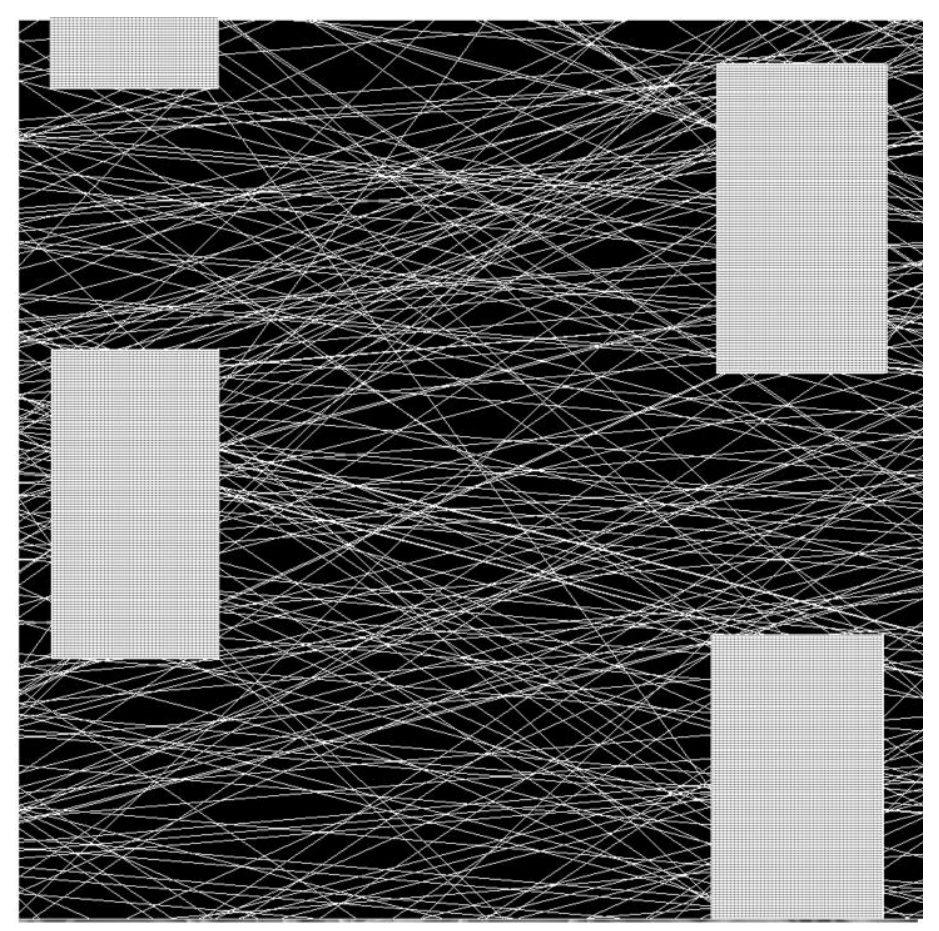

(b)

Fig. 4 


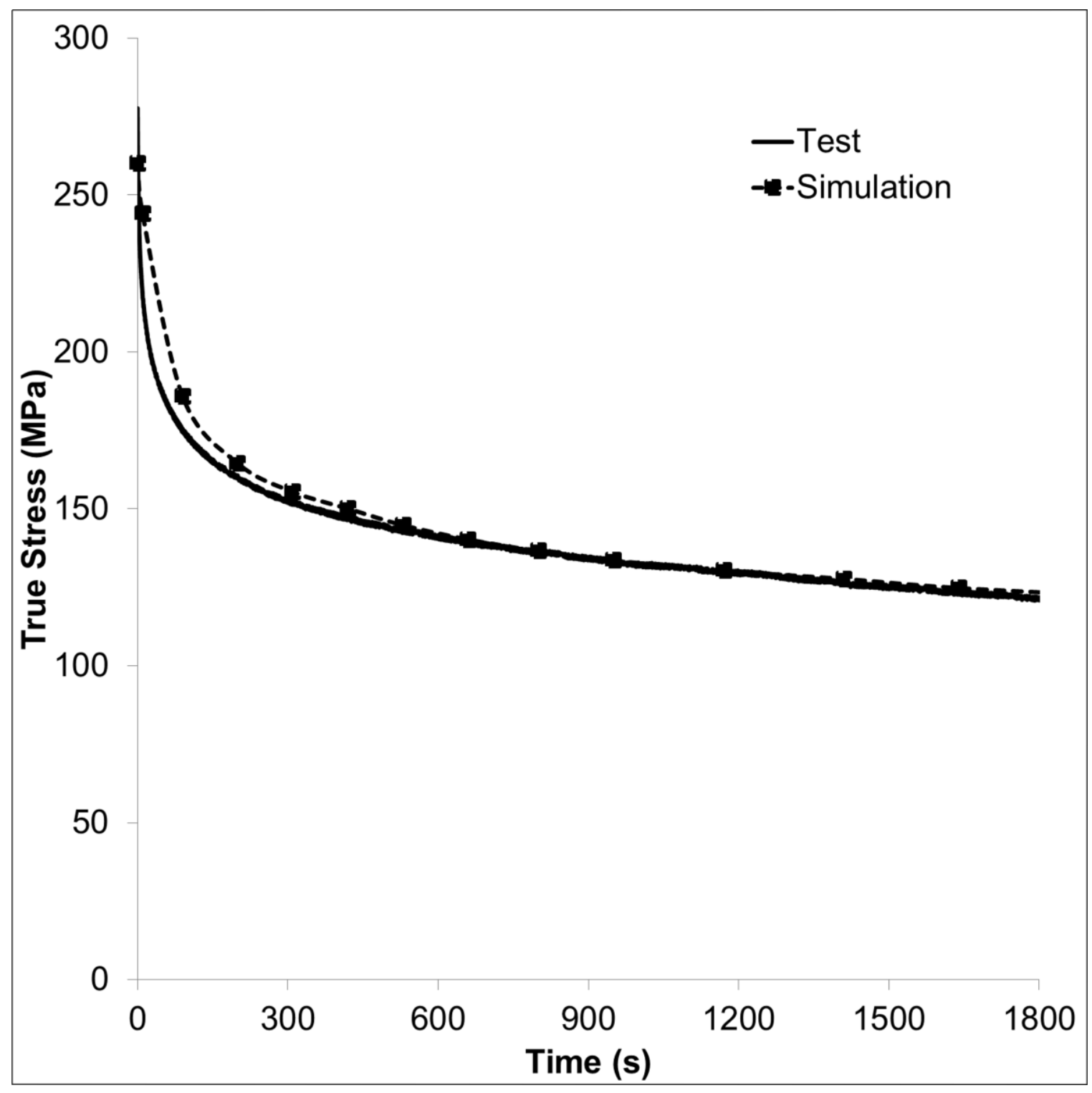

Fig. 5 

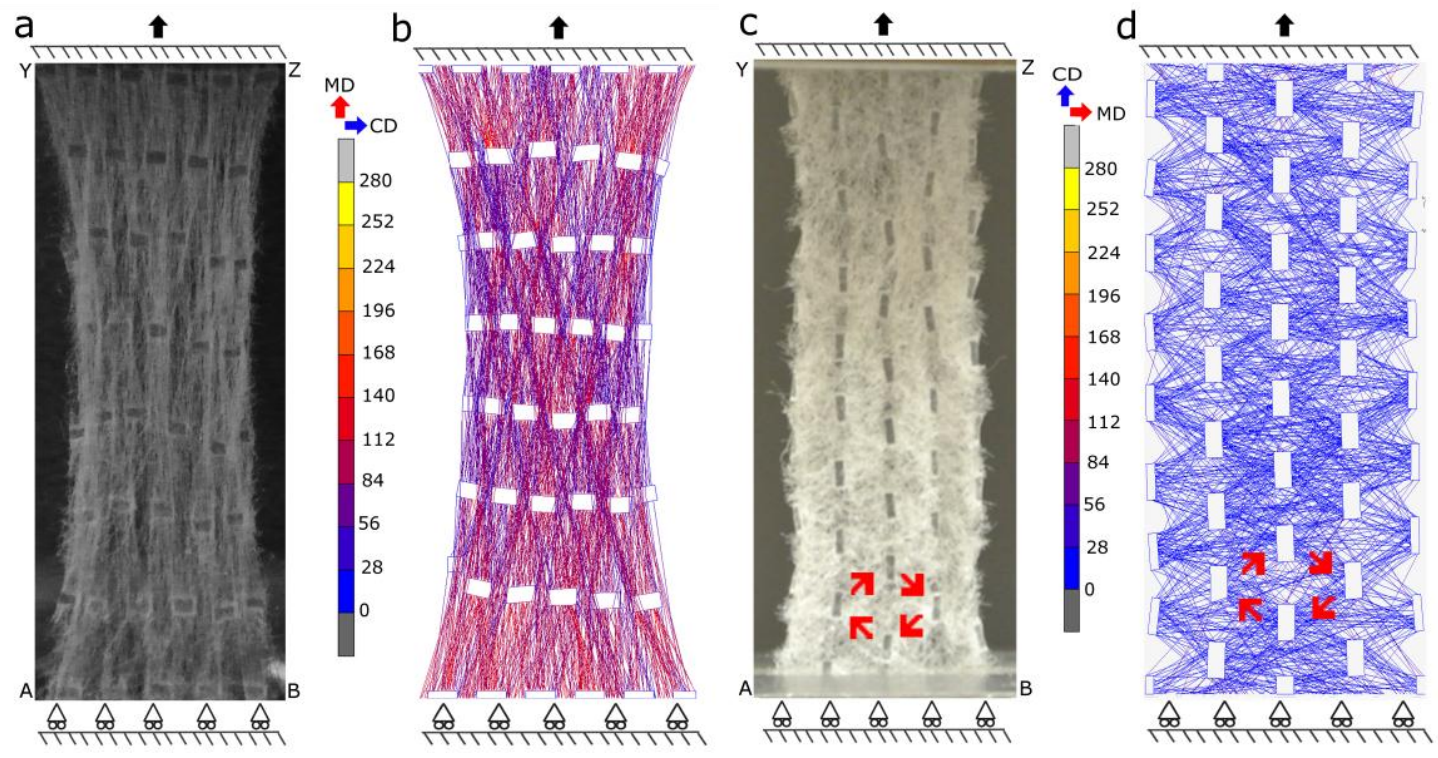

Fig. 6 


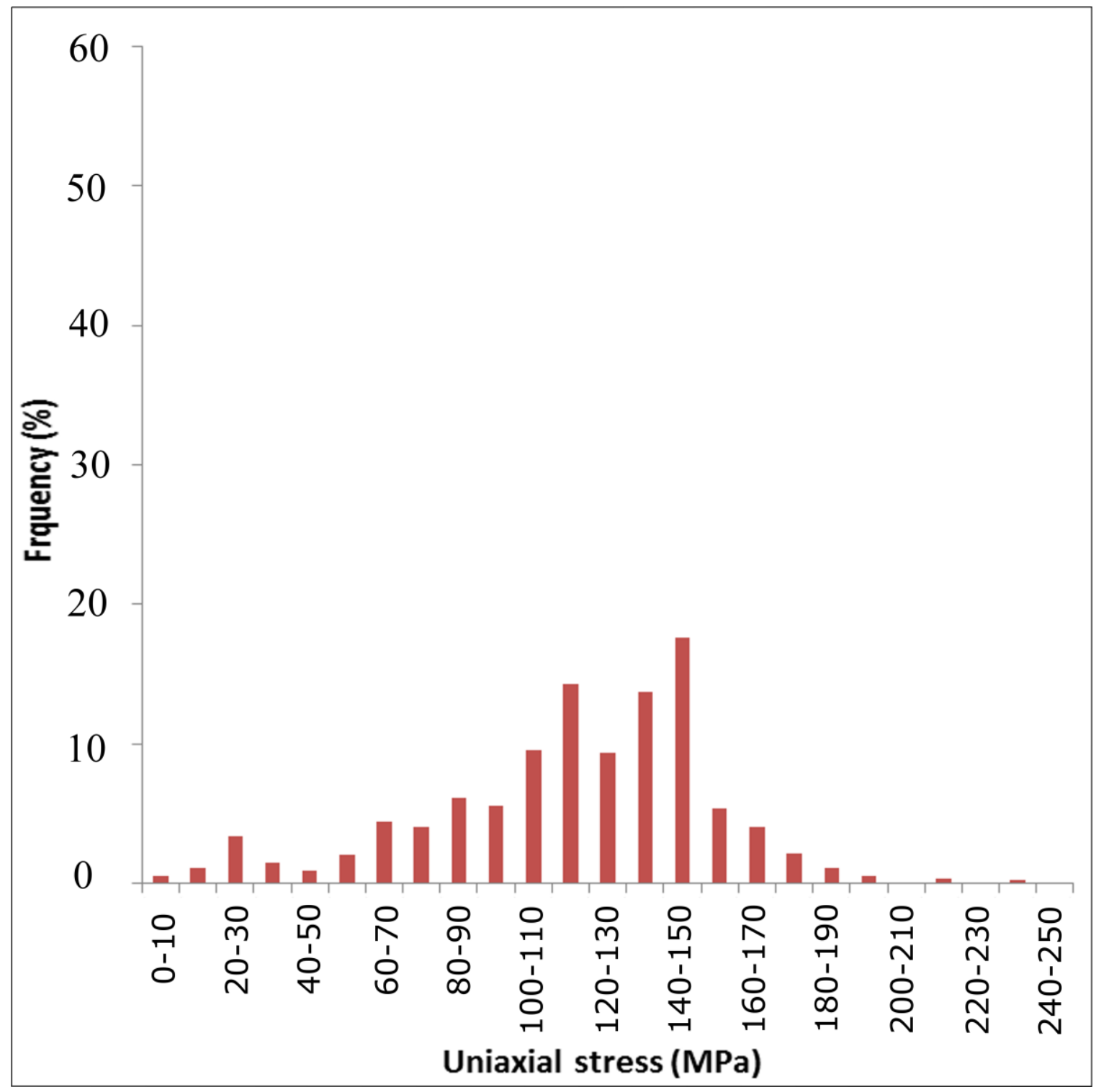

Fig. 7a 


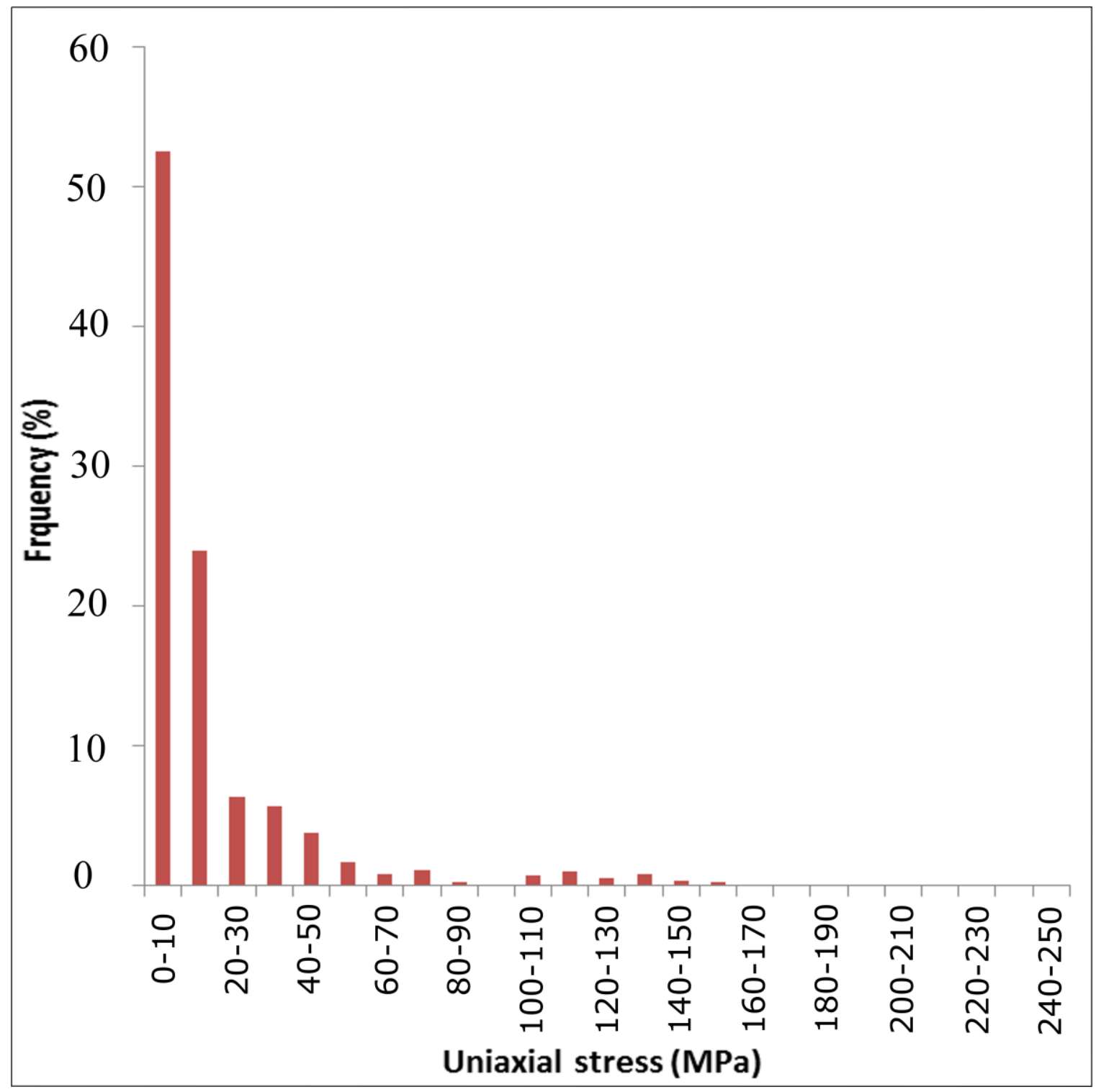

Fig. 7b 


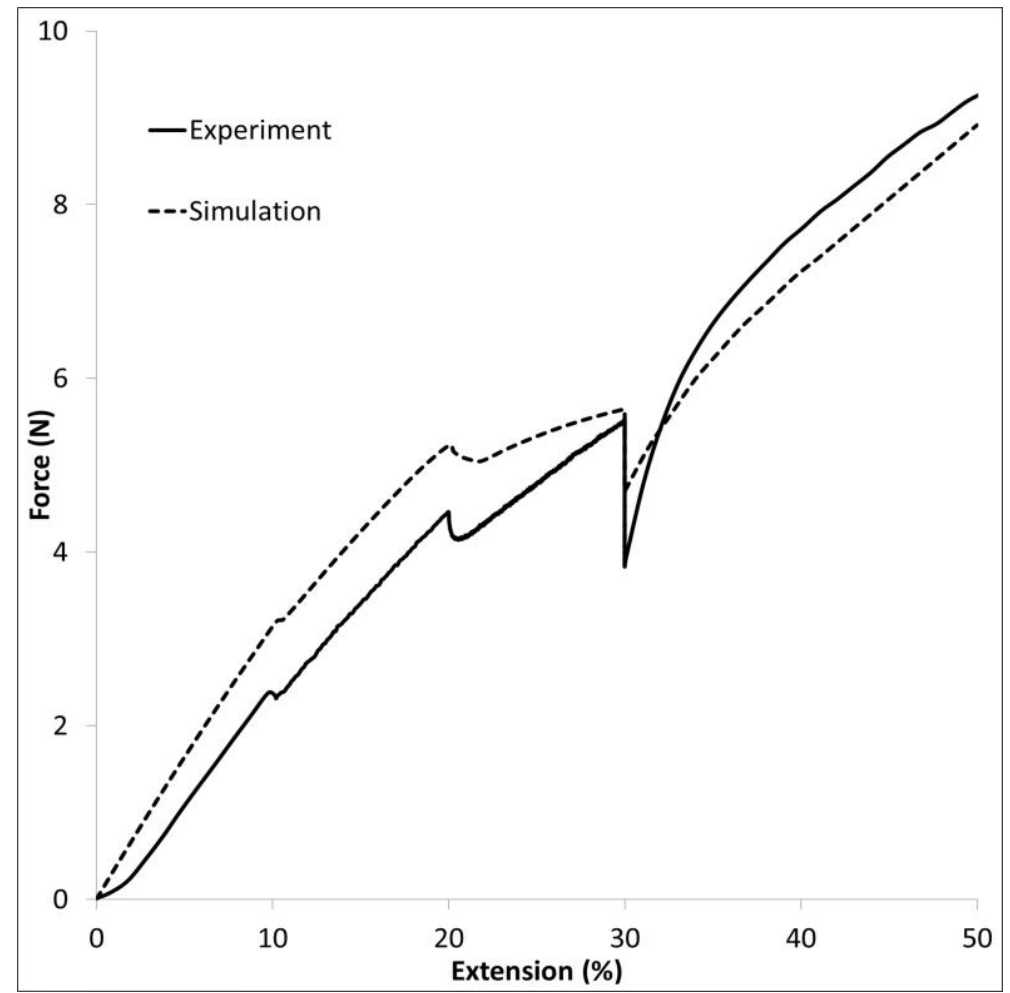

(a)

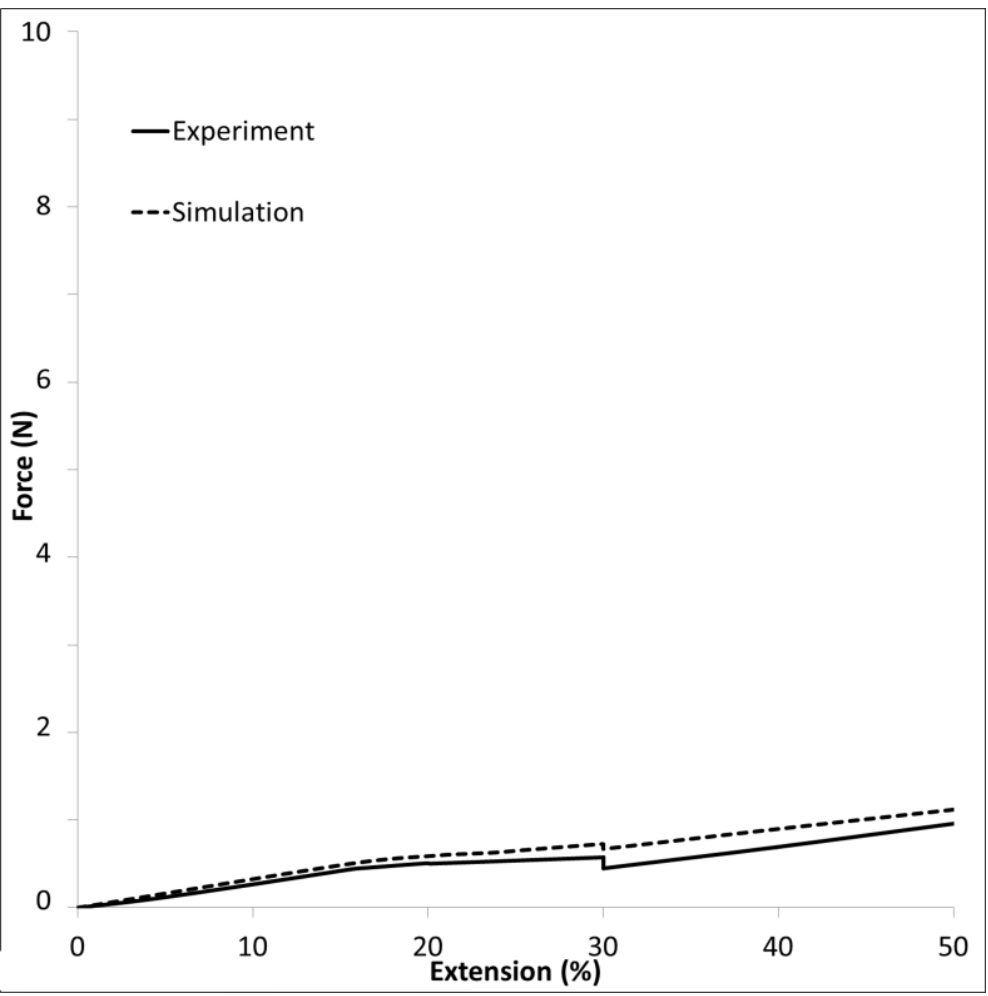

(b)

Fig. 8 